\title{
Beam squint and Stokes $V$ with off-axis feeds
}

\author{
Juan M. Uson and W. D. Cotton
}

\author{
National Radio Astronomy Observatory ${ }^{\star}, 520$ Edgemont Road, Charlottesville, VA 22903-2475, USA \\ e-mail: juson@nrao.edu
}

Received 4 February 2008 / Accepted 9 May 2008

\section{ABSTRACT}

\begin{abstract}
Radio telescopes with off-axis feeds, such as the (E)VLA, suffer from "beam squint" in which the two orthogonal circular polarizations sampled have different pointing centers on the sky. Its effects are weak near the beam center but become increasingly important towards the edge of the antenna power pattern where gains in the two polarizations at a given sky position are significantly different. This effect has limited VLA measurements of circular polarization (Stokes $V$ ) and introduced dynamic range limiting, wide-field artifacts in images made in Stokes $I$. We present an adaptation of the visibility-based deconvolution CLEAN method that can correct this defect "on the fly" while imaging, correcting as well the associated self-calibration. We present two examples of this technique using the procedure "Squint" within the Obit package which allows wide-field imaging in Stokes $V$ and reduced artifacts in Stokes $I$. We discuss the residual errors in these examples as well as a scheme for future correction of some of these errors. This technique can be generalized to implement temporally- and spatially-variable corrections, such as pointing and cross-polarization leakage errors.
\end{abstract}

Key words. techniques: image processing - techniques: interferometric - methods: data analysis

\section{Introduction}

The enhanced sensitivity of the EVLA will allow it to study sources much weaker than are currently observed. For frequencies at the lower end of the EVLA range this will generally require imaging the full primary beam of the antenna pattern, or at least those portions containing the stronger sources in the field in order to remove their sidelobes from the region of interest. In this regime, numerous effects of minor importance to observations of strong sources near the antenna pointing position must be understood and removed in order to allow the EVLA to reach its potential. "Beam squint" resulting from the off-axis arrangement of the feeds is one of these effects.

Many galactic objects have significant circular polarization in their emission. Without correction for the beam squint, the (E)VLA has strong off-axis instrumental circular polarization (Fig. 1) which masks any intrinsic circular polarization. Correction for the beam squint will result in a significant increase in the sensitivity of measurements of circular polarization.

(E)VLA observations are performed using dual orthogonal circular polarization (henceforth RCP and LCP). It has been known for quite some time that the antenna gain patterns formed on the sky for these two polarizations are not concentric, having a relative offset of $0.06 \pm 0.005$ of the antenna power FWHM; in reasonable agreement with a calculated value of 0.053 (Napier \& Gustincic 1977). The NVSS survey included careful measurements of the primary beam response of the VLA antennas at a frequency of $1.4 \mathrm{GHz}$. The beam squint was found to be $1.71^{\prime} \pm 0.02^{\prime}$ (Condon et al. 1998) corresponding to $0.055 \times$ FWHM (Fig. 1). Thus, for perfectly pointed observations, the gain in RCP and LCP towards a source located away from the pointing position will be modulated by up to $\sim 7 \%$ due to

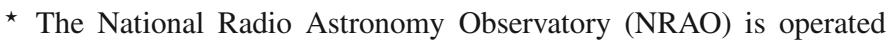
by Associated Universities Inc., under cooperative agreement with the National Science Foundation.

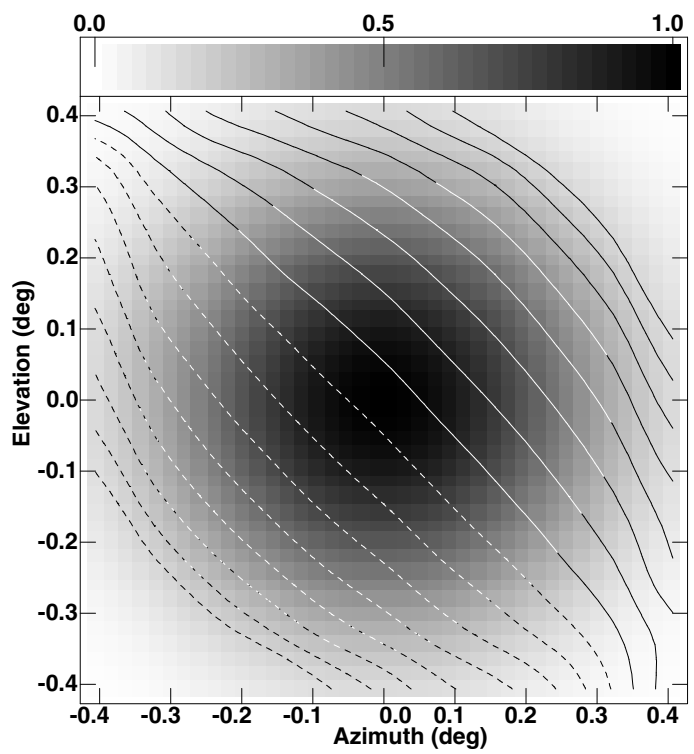

Fig. 1. The VLA primary antenna pattern as measured during the NVSS survey (Condon et al. 1998) at $1.4 \mathrm{GHz}$. The power pattern is shown in gray-scale with a scale bar at the top and contours are plotted at the $\pm 0.02, \pm 0.04, \ldots \pm 0.16$ levels of fractional Stokes $V$ (from the center outwards). Negative contours are dashed.

the Earth's rotation in approximately opposite sense below or above the nominal gain depending on the location of the source. The effect will be approximately equal in magnitude for the RCP and the LCP but of opposite sign and thus will largely cancel when estimating Stokes $I$; as long as both polarizations have been observed and preserved in the editing process. However, amplitude self-calibration might magnify the effect if corrections are determined separately for both polarizations as they will tend to counter the modulation of the strongest source affected by the 
beam squint. This can be prevented by preaveraging the RCP and LCP data prior to determining common amplitude correction factors.

In this paper, we discuss the beam squint in some detail, present an algorithm that corrects its effects and apply it to observations made with the VLA. We discuss as well the possible extension of this technique to a wider class of time and/or direction-dependent corrections. All data manipulation discussed in this report use the Obit package (Cotton 2008), (http://www.cv.nrao.edu/ bcotton/Obit.html).

\section{The (E)VLA beam squint}

Extensive studies of the polarization properties of "offset parabolas" have been performed since the early 1970s with detailed calculations describing the squint and cross-polarization properties of prime-focus offset parabolas (Chu \& Turrin 1973). Subsequent, more refined studies determined the polarization properties of a single-reflector parabola illuminated by a circularly-polarized feed located at the prime focus and whose axis is tilted by an angle $\theta_{0}$ with respect to the axis of the parabola. The resulting antenna primary beam is directed offaxis orthogonally to the plane that contains the axis of the parabola as well as that of the feed. The beam squint is given by (Duan \& Rahmat-Samii 1991):

$\theta_{\mathrm{S}}=\sin ^{-1}\left(\frac{\sin \theta_{0} \lambda}{4 \pi F}\right)$

where $\theta_{0}$ is the angle between the feed axis and the axis of the parabola, $\lambda$ is the wavelength of the observations and $F$ is the focal length of the parabola, $(F=9.0 \mathrm{~m}$ at the $(\mathrm{E}) \mathrm{VLA})$ and $\mp \theta_{\mathrm{S}}$ is the resulting beam squint of the (LCP, RCP) beam.

The dual reflector offset Cassegrain antenna was solved by defining an equivalent prime-focus parabola that is equivalent in performance to the wanted dual reflector Cassegrain antenna as it has the same co-polarized and cross-polarized radiation patterns (Rusch et al. 1990). It can be shown that the beam squint of such an antenna configuration is given by

$\theta_{\mathrm{S}}=\sin ^{-1}\left(\frac{\sin \left(\theta_{\beta}-\alpha\right) \lambda}{4 \pi F_{\mathrm{eq}}}\right)$

where $\alpha$ is the angle between the axis of the equivalent parabola and that of the feed and $\theta_{\beta}$ is the angle between the feed and the vertex of the off-axis, shaped subreflector and $F_{\text {eq }}$ is the equivalent focal length of the dual reflector. At the (E)VLA antennas, the corresponding values are $\alpha=59.8^{\circ}, \theta_{\beta}=1^{\circ}$ and $F_{\text {eq }}=59.1 \mathrm{~m}$ (Napier 1994). This reference discusses the derivation in some detail and shows that the beam squint is thus described by an inverse-sine function. However, the small-angle approximation is appropriate and thus the squint angle at the (E)VLA antennas scales quasi-linearly with wavelength. This is not necessarily the case with the FWHM of the primary beam response as this depends on the specific illumination due to the feed horns at each band. The description of the primary beam response as corresponding to uniform illumination of a $24.5 \mathrm{~m}$ circular aperture (appropriate at the VLA at $1.4 \mathrm{GHz}$ given the $25 \mathrm{~m}$ antenna diameter and the taper applied at the edge of the primary) leads to the $5.5 \%$ ratio discussed above. We have found that this ratio holds as well for the new (E)VLA $5 \mathrm{GHz}$ feed system (Uson \& Cotton, in preparation) but do not know at this time what the corresponding ratio at other frequencies of the (E)VLA will be. We intend to perform the relevant measurements in due course.

\section{Beam squint correction technique}

The beam squint can be characterized as a coupled offset of the pointing of the two orthogonally polarized beams. The antenna will be nominally pointed using the midpoint of these two beams giving an (opposite) effective pointing error in each polarization. In the following, the beam squint is treated as a polarizationdependent pointing error.

The beams are offset orthogonally to the line connecting the feed and the antenna center. Due to the alt-az mounts of the (E)VLA antennas, this offset will rotate on the sky with parallactic angle. Notice that by construction of the station pads, all (E)VLA antennas have the same parallactic angle at any given time even in the extended configurations.

The effect of beam squint on a given source depends on its location in the field of view so, in general, no operation on the uv data can remove the effects of beam squint over the whole field, although an approximation can be attempted through construction of a suitable unitary operator (e.g. Bhatnagar et al. 2008). However, such a procedure encounters problems on fields with significant emission from sources located near the nulls of the primary beam. The effects of beam squint can be included whenever the instrumental response to a model (e.g., a set of CLEAN components) is computed. In the algorithm described here, this is done using a discrete Fourier transform where the instrumental response for each visibility is computed for each component and then summed over components. The flux density of each component is modified by the ratio of the antenna gain in the nominal pointing direction to the actual, beam squint dependent, gain. This is done independently for each polarization. As the beam squint rotates with parallactic angle, this flux density correction is time as well as direction dependent.

There are two instances where this interferometer response model is used. The first is in a visibility-based ("SchwabCotton", Schwab 1983; Cotton 1999) CLEAN in which a set of components is initially located by a major cycle of the "Clark", Clark (1980) CLEAN followed by an accurate model calculation allowing the effects of those components to be removed from the visibility data and a new residual image derived from the residual visibility data. Thus, errors in the initial image due to inadequacies in the initial response model are corrected as deconvolution progresses.

The other use of the instrumental response model is in the self-calibration step in which the visibilities are divided by the corresponding instrumental response to the sky model to produce a dataset equivalent to observations of a point source with a perfect instrument. Using an accurate beam-squint model allows the removal of the effects of beam squint from the resultant gain solution.

If an image in Stokes $V$ is desired, then Stokes $I$ must be imaged first and the Stokes $I$ model subtracted from the data with beam squint corrections applied. This should remove the instrumental circular polarization from the data. Subsequently, the Stokes $V$ image can be derived and deconvolved without further beam squint corrections. Instrumental leakage terms (the socalled D-terms) have been ignored in the procedure discussed in this paper, but could be incorporated if necessary as long as such leakage terms were determined with sufficient precision.

\subsection{CLEAN model for beam squint corrections}

In the normal model calculation, a list of CLEAN components is kept and, for each visibility measurement, the instrumental response to each component is calculated with the total 
instrumental response obtained by summing over all components. To apply the beam squint correction, the time- and polarization-dependent antenna gain corrections are used to adjust the component flux density for the effects of beam squint on the antenna power pattern. To do this, the antenna voltage gain relative to the nominal pointing is determined for the RCP and LCP beams in the direction of each CLEAN component and the interferometric (power) gain correction is obtained as the product of the antenna voltage gain corrections. These gain corrections are updated whenever the observing parallactic angle changes by one degree. Of course, this interval could be shortened if necessary.

A mixed array such as the VLA+EVLA adds another complication as the feed placement is different for the two types of antennas (Cotton \& Uson 2007a). In this case, the right- and lefthand voltage gain ratios are computed separately for VLA and EVLA antennas and the appropriate voltage gains are applied to the component flux density for each baseline.

The implementation described above assumes that all antennas observe the sky at the same parallactic angle. This is indeed the case at the (E)VLA as care was taken during the construction of the VLA to build the antenna mounts to a common horizon. For arrays such as the VLBA, parallactic angle will differ from antenna to antenna and an implementation for such an array would need to maintain lists of voltage gain corrections for each antenna. This is straightforward and simply adds to the procedure some bookkeeping as well as computational overhead.

If large numbers of CLEAN components are involved, this technique is expensive in computer cycles and memory. A simple expedient procedure is to "compress" the list by summing the flux density of all components derived from a given image grid cell and using at most one component per cell. An additional benefit of summing the components in each cell is the reduction of numerical round-off error in calculating the model response.

\subsection{Beam squint gain corrections}

This beam squint correcting algorithm has been implemented in the Obit package as task "Squint." The pointing offset in antenna coordinates due to the beam squint is orthogonal to the line from the feed to the antenna center with its value given by Eq. (2) using the values given by Napier (1994).

Thus, the offset for each polarization at the (E)VLA is given by

squint $=\mp 237.56 \times \lambda\left(\frac{\operatorname{arcsec}}{\mathrm{m}}\right)$

where $\lambda$ is the wavelength $(\mathrm{m})$. The value of squint is negative for LCP and positive for RCP. The offsets in RA and Dec are then:

$\mathrm{d} x=$ squint $*(-\sin ($ feedAngle $-\chi))$

$\mathrm{d} y=$ squint $*(\cos ($ feedAngle $-\chi))$

where feedAngle is the orientation of the feed on the feed circle, $\chi$ is the parallactic angle and the sign of squint is appropriate for the polarization (Cotton \& Uson 2007a).

For a given CLEAN component, the distance from the pointing position corrected for beam squint is given by:

$$
\begin{aligned}
\mathrm{d}(x, y)= & \cos ^{-1}\left\{\sin \left(\delta_{\text {off }}\right) \sin \left(\delta_{\text {Pnt }}\right)\right. \\
& \left.+\cos \left(\delta_{\text {off }}\right) \cos \left(\delta_{\text {Pnt }}\right) \cos \left(\alpha_{\text {off }}-\alpha_{\text {Pnt }}\right)\right\}
\end{aligned}
$$

where $\alpha_{\mathrm{Pnt}}$ and $\delta_{\mathrm{Pnt}}$ are the RA and Dec of the antenna pointing, $\alpha_{\text {off }}=\alpha_{\mathrm{CC}}+x, \delta_{\text {off }}=\delta_{\mathrm{CC}}+y$, and $\alpha_{\mathrm{CC}}$ and $\delta_{\mathrm{CC}}$ are the RA and Dec of the component.
For frequencies above $1 \mathrm{GHz}$, the antenna pattern is approximated above the $5 \%$ level by a Jinc function ${ }^{1}$ which is in turn approximated using Sect. 9.4.4 in Abramowitz \& Stegun (1964):

$P(x, y)=4.0\left(0.5+\sum_{n=1}^{6} c_{n} u^{n}\right)^{2}$

where

$u=\left\{1.496 \times 10^{-9} \times\left(\frac{25.0}{d_{\mathrm{ant}}}\right) \times \mathrm{d}(x, y) \times v\right\}^{2}$

and $c_{1}=-0.56249985, c_{2}=0.21093573, c_{3}=-0.03954289$, $c_{4}=0.00443319, c_{5}=-0.00031761, c_{6}=0.00001109$ and $d_{\text {ant }}$ is the effective antenna diameter in meters with $d_{\text {ant }} \sim 24.5 \mathrm{~m}$ at a frequency of $1.4 \mathrm{GHz}$ (Condon et al. 1998). The voltage gain for each antenna and polarization is then:

voltage_gain $=\sqrt{P(x, y)}$.

As described above, the correction assumes a single azimuthallysymmetric antenna pattern describable by a 1-D function. A single 2-D antenna pattern could be readily incorporated as a lookup table. Antenna-specific beam patterns require maintaining separate lists of component gains for each antenna. In the case of a large number of components, this method can be time consuming. Therefore, in the implementation described here, a threshold is defined that specifies the cutoff between the levels that are processed using the high accuracy squint correction model and those that are not. If the purpose of the application of this technique is solely to improve the dynamic range in Stokes I by reducing the artifacts arising from the brightest emission in the field, it is not necessary to compute a highly accurate model for components corresponding to regions of weaker emission. By restricting the accurate model calculation to the portion of the model representing the brightest emission and allowing faster, but less accurate calculations for the weaker emission, the expense of this algorithm can be drastically reduced. Because extended, low-level emission can produce a coherent error pattern it is important to keep this threshold rather low. In addition, if Stokes $V$ imaging is desired, the beam squint correction must be applied to the entire Stokes $I$ model. During CLEANing, whenever the peak in the initial residual image at the start of a major cycle exceeds the threshold value, the high accuracy model is required for all components. When the peak residual drops below this threshold, a lower accuracy model calculation is allowed if it is deemed to be faster. For self-calibration or other cases where the total CLEAN model is known, the components corresponding to a given image cell are combined and those whose flux densities exceed the threshold are processed with the high accuracy model, whereas those below the threshold may be processed with the faster method. In all cases, components corresponding to a given cell are combined before the model calculation. A nonzero threshold for using the more accurate calculation might limit the dynamic range because of its effect on the amplitude self-calibration; a lower threshold leads to higher accuracy, although at the expense of execution time.

\subsection{Correction of individual antenna patterns}

Detailed differences among antennas will cause differences in their radiation patterns. For extremely high dynamic range,

1 The arbitrary cutoff at the $5 \%$ response is due to our lack of precise knowledge of the (E)VLA primary beam response at low levels; we are investigating ways to extend the squint correction to lower levels. 

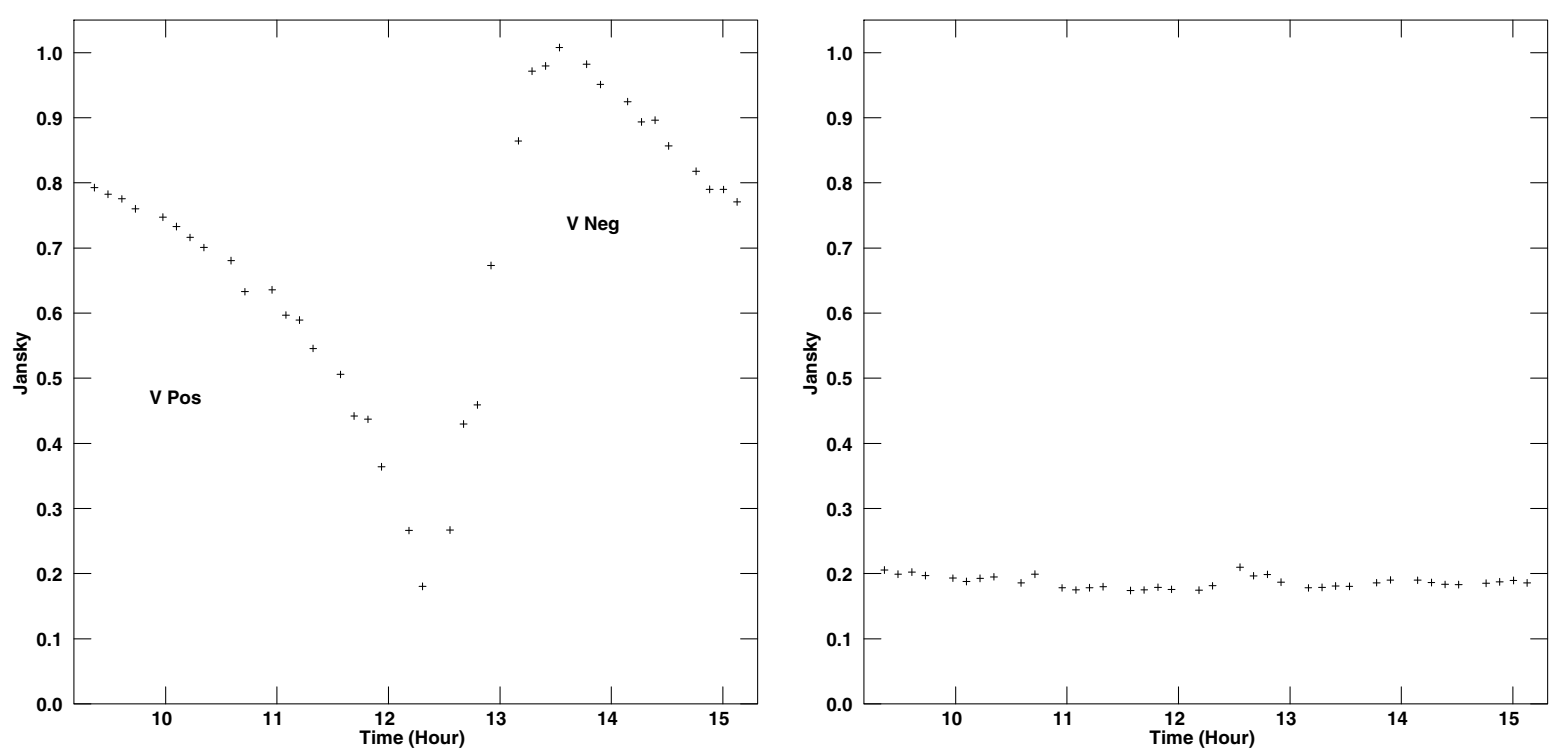

Fig. 2. Time averaged $1.4 \mathrm{GHz}$ Stokes $V$ for observations with $3 \mathrm{C} 84$ located at the half-power point westward of the pointing center. The left panel shows uncorrected data, the minimum is located where the Stokes $V$ changes sign; positive and negative regions of Stokes $V$ are indicated. The right panel shows the corresponding data after subtraction of the beam-squint corrected Stokes $I$ model. The amplitude bias of the individual measurements is $\sim 200 \mathrm{mJy}$.

wide-field observations corrections for these differences among antennas will be necessary. This case is similar to the beam squint problem in that time-variable and directional gain corrections are needed. A straightforward modification of the technique described in this paper would allow correcting for detailed antenna patterns, so that errors due to cross-polarization leakage could be corrected in the manner described here.

\subsection{Correction of antenna pointing errors}

As mentioned in the introduction, in the absence of pointing errors, the effects of beam squint on RR and LL correlations will be approximately equal but of opposite sign. Thus, in this case, imaging of Stokes $I(0.5 *(\mathrm{RR}+\mathrm{LL}))$ will be largely, but not completely, insensitive to beam squint. However, in the presence of pointing errors, the magnitude of the beam squint on RR and LL will be different and will not be eliminated by imaging Stokes $I$, or by the technique described here without additional pointing corrections.

At this writing, global, antenna-based pointing errors (i.e., collimation errors) can be readily incorporated as our algorithm treats the beam-squint as a coupled $(R, L)$ pointing error. If postobservation corrections of time-variable antenna pointing errors could be determined, a straightforward modification of this technique could be applied. Effective component gains for each antenna and polarization could be kept, including the antenna pointing errors as well as the beam squint. A time and antenna dependent addition to the beam squint position offsets would be used in computing the effective component gains. This would simply require some bookkeeping and involve extra, straightforward computations.

\section{Verification using actual data}

\subsection{Example 1: off-axis observations of $3 C 84$}

The beam squint algorithm was tested on VLA B configuration observations of $3 \mathrm{C} 84$ in which the source was alternatively located on-axis, at the $1 / 2$ power of the antenna beam (westward) and at the $1 / 2$ power and $1 / 3$ power of the antenna beam (southward). Observations were made in both the 1.4 and $5 \mathrm{GHz}$ bands in spectral line mode, recording $15 \times 390 \mathrm{kHz}$ channels. The observations were made in July and August 2006. The data were amplitude, phase and bandpass calibrated in the normal fashion using the on-axis pointings with 2-point interpolation of the gain solutions (amplitude, phase) to the other pointings

The data were processed twice, once without and once with beam squint correction applied but otherwise processing was the same. All imaging used the "autocenter" technique (Cotton \& Uson 2007b), to improve the dynamic range. A single iteration of phase-only self-calibration with a solution interval of $30 \mathrm{~s}$ was followed by a single iteration of amplitude and phase selfcalibration with a 2-min solution interval. Self-calibration solutions were determined for an average of right- and left-hand circular polarization as without beam squint corrections, the independent right- and left-hand amplitude self-calibration would "correct" the data for 3C 84 at the expense of the rest of the field. In the following paragraphs, we discuss the observations of $3 \mathrm{C} 84$ at a frequency of $1.4 \mathrm{GHz}$ with the source observed at the (westerly) half-power location of the antenna primary beam.

A convenient way to see the effects of beam squint and to test the efficacy of the correction is to examine a plot of the timeaveraged Stokes $V$ amplitudes averaged as well over baselines and spectral channels (Fig. 2, left panel). The instrumental polarization is fixed to the antenna and will rotate on the sky with parallactic angle causing a time-variable Stokes $V$ signal in the averaged data. Since the source is not located at the phase center, this averaging must be done over amplitudes and therefore will suffer from a significant amplitude bias. Also, since the figure shows only the amplitude of the averaged visibilities, both positive and negative Stokes $V$ appear with positive sign with a dip in the curve where the instrumental response changes the sign of Stokes $V$.

Each data set was imaged using these corrections to derive a Stokes $I$ model which was then subtracted from the data 

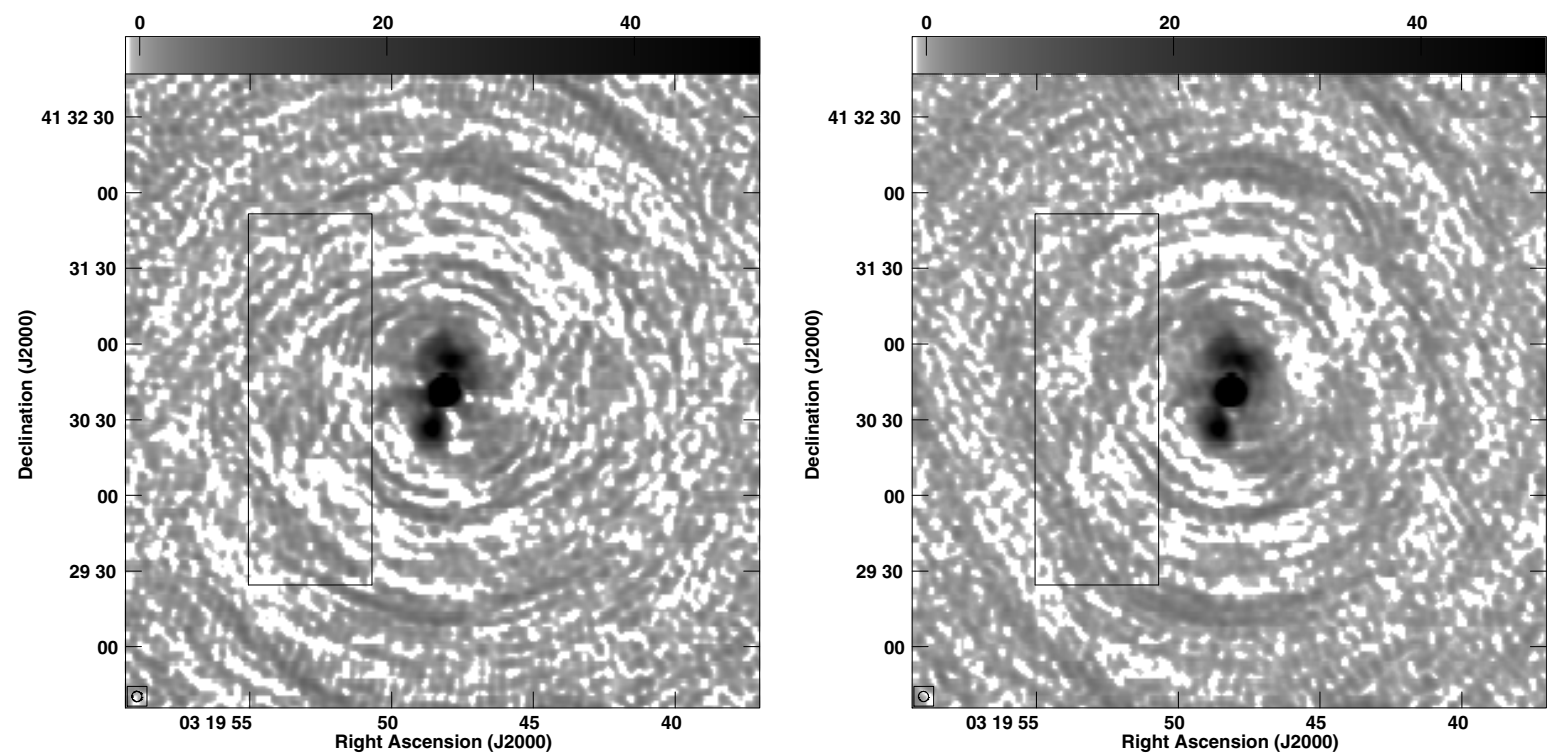

Fig. 3. Left: example 1. Stokes $I$ in reverse gray-scale of the region around $3 \mathrm{C} 84$ imaged with no beam squint correction. The image is displayed with a square root transfer function and the scale is given by the wedge (in $\mathrm{mJy} / \mathrm{beam}$ ) at the top. The box gives the region over which the rms was determined. Right: as left but with beam squint corrections.
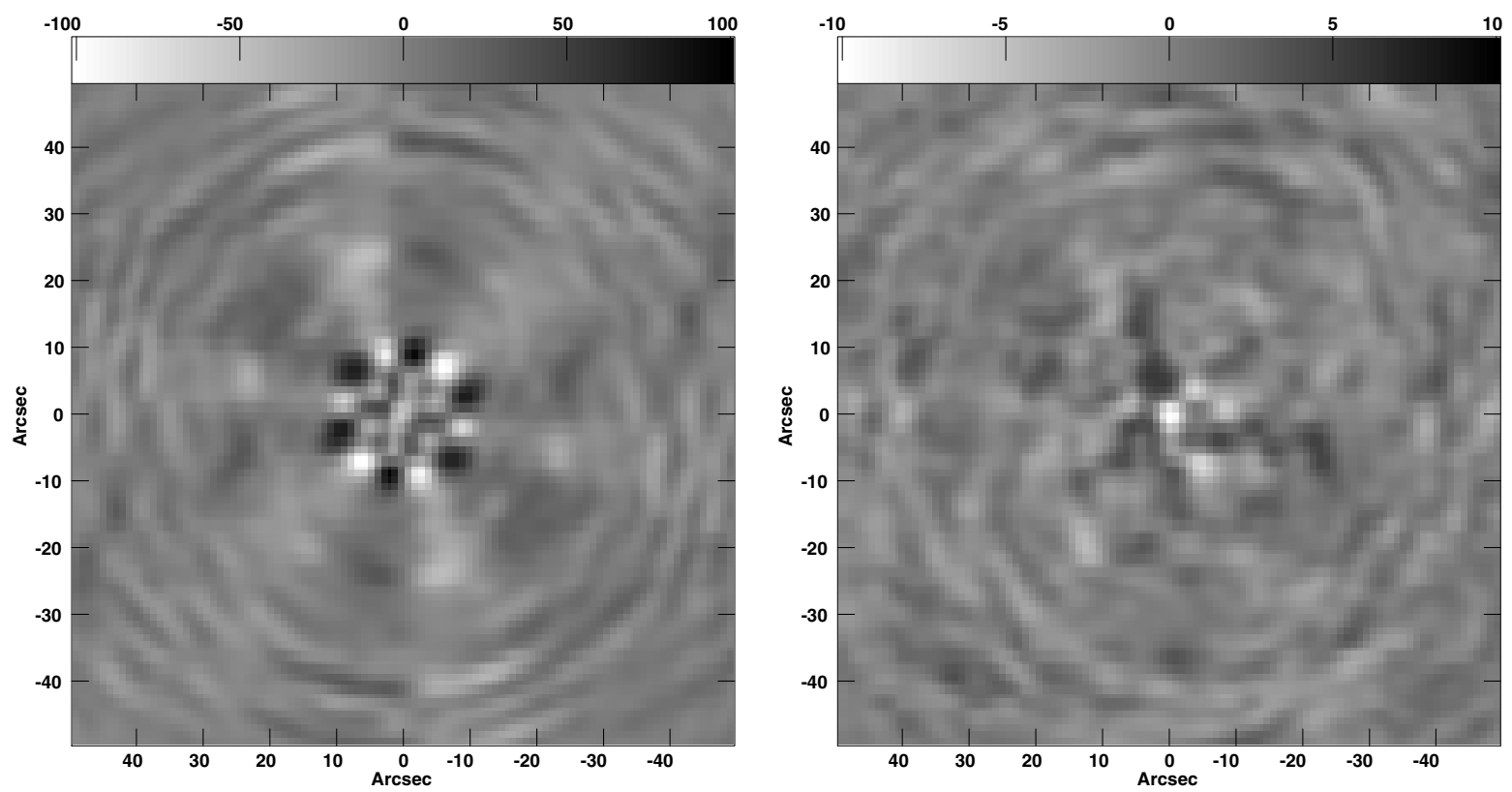

Fig. 4. Left: example 1. Stokes $V$ in reverse gray-scale of the region around $3 \mathrm{C} 84$ imaged with no beam squint correction shown with a linear display of -100 to $100 \mathrm{mJy} /$ beam. The scale is given by the wedge at the top. Right: as left but with beam squint corrections. Notice the finer scale displaying the range of values -10 to $10 \mathrm{mJy} /$ beam.

after performing the squint correction. A time-averaged Stokes $V$ plot (Fig. 2, right panel) will show the extent to which the procedure has worked. The $200 \mathrm{mJy}$ level in Fig. 2 corresponds to the amplitude bias of the individual time and spectral samples. This figure shows that the beam squint has been corrected to the level expected from the amplitude bias and within the scatter expected from the noise in the individual measurements. Similar results were obtained for the $5 \mathrm{GHz}$ data.

The Stokes $I$ images are shown in Fig. 3 with a squareroot stretch of the lower pixel values in the image (peak 12.2 Jy/beam). The image made without beam-squint correction shows more residual artifacts than the image with the correction applied. To compare the two images quantitatively, the rms noise was computed in identical boxes which are shown in Fig. 3. The $\mathrm{rms}$ in the image without correction was $1.47 \mathrm{mJy} / \mathrm{beam}$ whereas it was $1.16 \mathrm{mJy} / \mathrm{beam}$ in the image made with the squint correction, a reduction of the level of artifacts by $21 \%$.

Figure 4 compares the Stokes $V$ results. Since the data included observations over a range of parallactic angles, much of the instantaneous instrumental circular polarization is washed out. Nevertheless, the image made without squint correction shows residual errors that are one order of magnitude larger than those in the squint-corrected image which is displayed using only the inner $10 \%$ of the pixel range of the uncorrected image. In the uncorrected image, the maximum and minimum values are well off the source but the maximum value in the corrected 
image is on-source. However, this analysis becomes confused as the core in this source is known to show circular polarization above the level shown in Fig. 4. Since the data were calibrated using 3C 84 and assuming no circular polarization, the polarization calibration is incorrect. Unfortunately no other calibrators were included in the observations. The off-source noise levels computed using the same region as in the Stokes $I$ images are $7.9 \mathrm{mJy} /$ beam and $0.94 \mathrm{mJy} /$ beam for the un-corrected and corrected images (respectively).

In this test, the beam squint correction leads to a significant improvement to the quality of the Stokes $I$ image and to a very large improvement in the Stokes $V$ image.

\subsection{Example 2: imaging of full-track observations}

Our second example is a deep, full-track (two passes) $1.4 \mathrm{GHz}$ H I observation of the field of the superthin galaxy IC 2233 which contains significant background continuum emission totaling $\sim 1.1 \mathrm{Jy}$ (observed, i.e. before correction for the attenuation of the primary beam) which is dominated by two bright "4C" sources (4C+46.17 at J081430.4+455639.4 (henceforth the "upper-left" source) and 4C+45.15 at J081242.0+453651.3 (hereafter the "lower-right" source). The sources are observed with flux densities of $0.87 \mathrm{Jy}$ and $0.18 \mathrm{Jy}$, uncorrected for the $80 \%$ and $34 \%$ (respectively) attenuation of the primary beam. These sources limit the dynamic range significantly. The data were obtained with the VLA in C configuration on 2000 May 28-29 during a period of solar maximum. A full analysis of the spectral observations has been published elsewhere (Matthews $\&$ Uson 2008). We discuss here the imaging of the continuum emission with and without the squint correction as well as the limiting factors and residual errors in the squint-corrected images.

The observations were made in two overlapping frequency bands, each with $63 \times 24.4 \mathrm{kHz}$ channels, which allowed for 6 line-free spectral channels from the low-frequency side of the first (lower frequency) band and 14 line-free channels from the high-frequency side of the second (higher frequency) band, after discarding 6 channels on each end that were contaminated by ghost images from the two strong " $4 \mathrm{C}$ " sources in the field (Uson 2007). The remaining line-free channels $(6+14)$ were processed using conventional methods to make the images shown on the left-hand side of Figs. 5 and 6. Phase-only self-calibration was performed on a time scale of $20 \mathrm{~s}$ with the corrections applied to the data, followed by amplitude and phase self-calibration performed on 5-min time intervals after pre-averaging the RCP and LCP data with these corrections applied to both polarizations. The Stokes $I$ image has an rms noise level of $150 \mu \mathrm{Jy} /$ beam and quite significant residual sidelobe patterns surround the two " $4 \mathrm{C}$ " sources. The variable squint results in a modulation of the apparent Stokes $V$ signal as a function of time. Thus, the uncorrected Stokes $V$ image shown in the lefthand panel of Fig. 6 shows strong sidelobes that cannot be deconvolved as they correspond to a variable "source" that does not satisfy the average deconvolution equation expected from the synthesized beam that corresponds to the full uv-coverage. The rms noise is $190 \mu \mathrm{Jy} /$ beam but the noise distribution is clearly non-Gaussian.

We have applied our squint-correcting procedure to these data. The procedure included automatic selection of cell-size and imaging grid of "fly's-eye" fields and outliers, as well as "autocentering" (Cotton \& Uson 2007b), to improve the dynamic range. The procedure incorporates phase-only selfcalibration (20-s intervals) followed by amplitude and phase selfcalibration (5-min intervals). It is worth noting that the selfcalibration operations used about one half of the number of degrees of freedom used with the conventional analysis because both bands were combined into one 86-channel "band" before applying our algorithm. The rms noise in the resulting images is $110 \mu \mathrm{Jy} /$ beam for the Stokes $I$ image and $105 \mu \mathrm{Jy} /$ beam for the Stokes $V$ image.

The results are shown in the right-hand panels of Figs. 5 and 6. Although the Stokes $V$ image is largely consistent with noise with peak values at $\pm 4.7 \sigma$, a faint pattern of residual sidelobes is visible surrounding the position of the upper-left " $4 \mathrm{C}$ " source. Some residual sidelobes to the second-strongest source remain in the Stokes $I$ image as well. This lower-right source is located at a point of steep gradient in the primary beam response and is thus significantly affected by collimation and pointing errors. We believe that such errors are likely to be responsible for our failure to correct this image in full. Of course, any uncorrected systematic effects will lead to errors in the selfcalibration solutions. Despite the presence of such residual systematic errors, the Squint algorithm yielded an increase in dynamic range by more than one order of magnitude in Stokes $V$ and a decrease of the rms noise in the Stokes $I$ image of $\sim 27 \%$.

\section{Conclusions}

Taking advantage of the EVLA's increased sensitivity at lower frequencies ( $15 \mathrm{GHz}$ and below) will generally require imaging and deconvolving the entire primary beam, or at least those portions containing sources. Beam squint can produce artifacts well above the noise level. Other interferometers with off-axis feeds, including focal plane array feeds, will share this problem

This paper describes a technique for correcting radio interferometric observations for the effects of beam squint. The technique is a modification of the visibility-based CLEAN in which the Stokes I image is made in the usual way, but when the image model is subtracted from the data, corrections are made such that the model subtracted includes the effects of beam squint. Thus, any errors incurred in the initial imaging are corrected through iterative imaging and accurate model subtraction. Selfcalibration is incorporated into the procedure in two steps with phase-only selfcalibration performed first, subsequently followed by amplitude and phase selfcalibration.

We have performed several tests of an implementation of this technique in the Obit task Squint. In all cases, careful initial calibration is critical. Observations of the strong source 3C 84 were made with the source at various locations in the antenna beam pattern resulting in strong instrumental circular polarization. Both 1.4 and $5 \mathrm{GHz}$ test data sets were examined and we found that this technique corrects the instrumental circular polarization due to the beam squint to a level that is limited by other effects such as pointing errors and insufficient knowledge of the primary beam.

We have presented detailed examples of imaging of several observations. Overall, applying the beam squint correction lowered residual artifacts in the vicinity of strong sources by more than $20 \%$ and led to very substantial improvements to the Stokes $V$ images. Our second example demonstrates the behavior of the algorithm on an observation that requires high dynamic range. Here, artifacts remain that can be ascribed to collimation and pointing errors. It would be straightforward to extend this technique to correct for individual antenna patterns and known (i.e., determined elsewhere) antenna pointing errors.

We are investigating the primary beam and pointing parameters of the (E)VLA antennas at several frequency bands and hope 

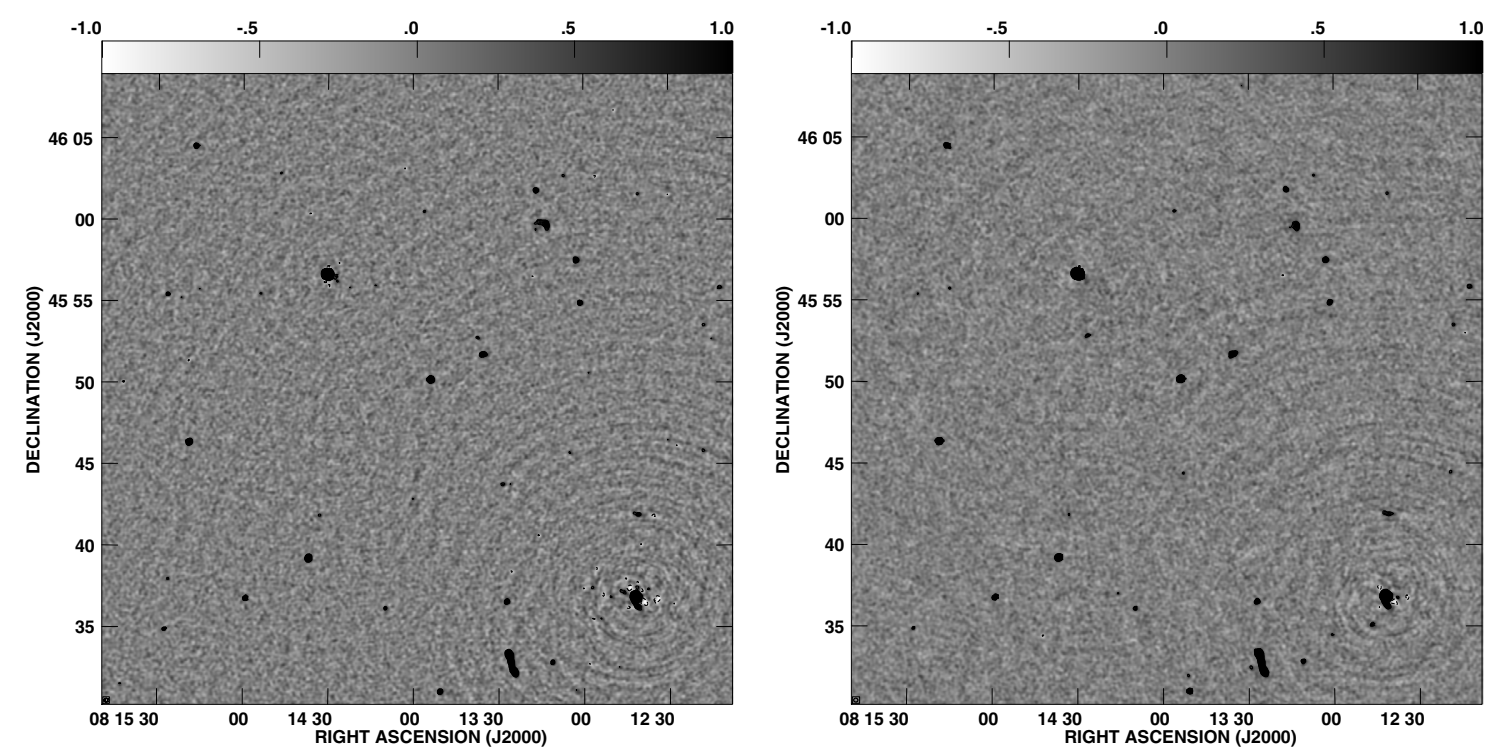

Fig. 5. Left: example 2. Stokes $I$ in reverse gray-scale of the field of IC 2233 imaged with no beam squint correction. Notice the deconvolution errors due to the two " $4 \mathrm{C}$ " radio sources. The image is displayed with the scale given by the wedge (mJy/beam) at the top. The rms noise is $\sim 150 \mu \mathrm{Jy} /$ beam. Right: as left but with beam squint corrections. The noise is reduced to $\sim 110 \mu \mathrm{Jy} / \mathrm{beam}$. Some artifacts remain that are centered on the " $4 \mathrm{C}$ " source on the lower-right side.
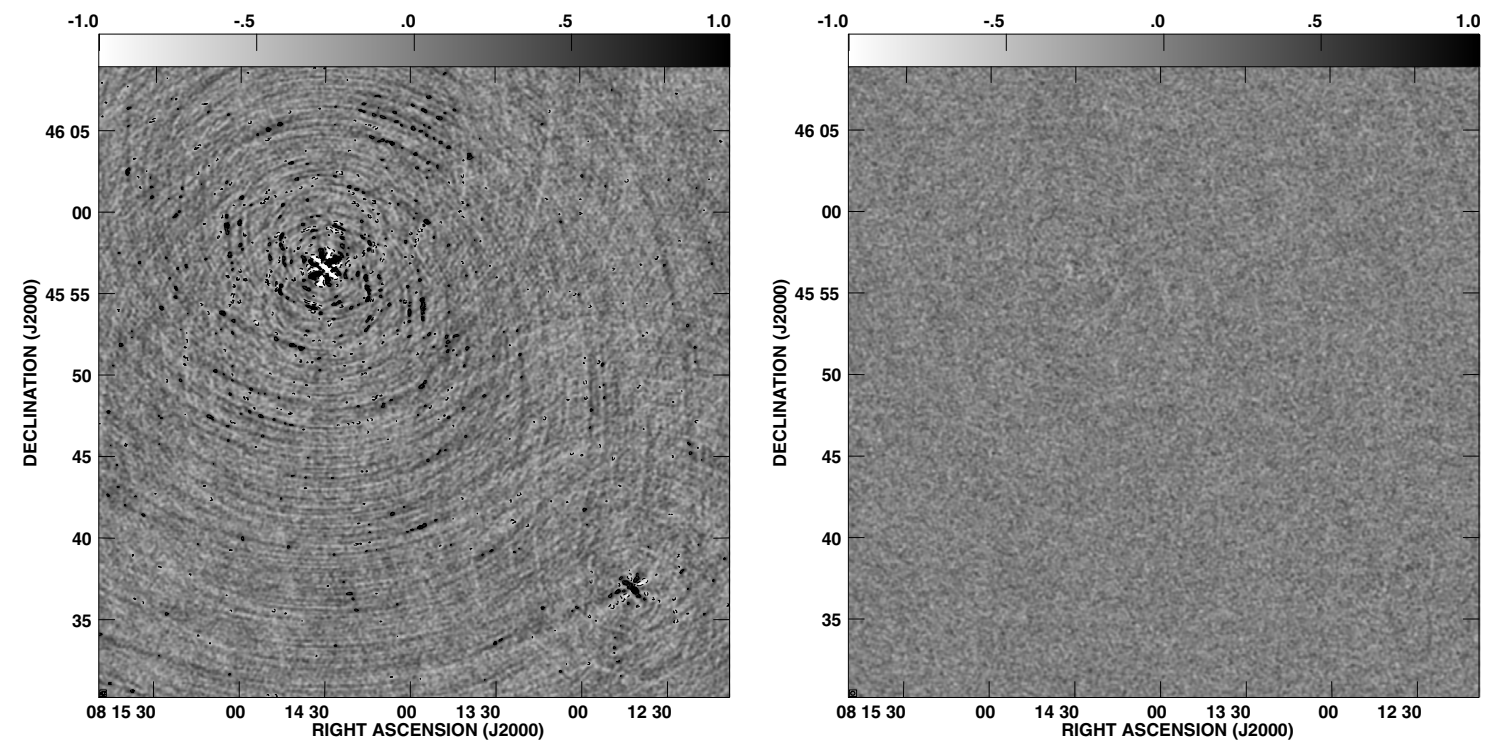

Fig. 6. Left: example 2. Stokes $V$ in gray-scale of the field of IC 2233 imaged with no beam squint correction. Notice the artifacts due to the variable Stokes $V$ "signal" at the position of the two " 4 C" sources induced by the Earth's rotation of the (squinted) primary beam response with respect to the sky. This error pattern determines the RMS noise value of $190 \mu \mathrm{Jy} / \mathrm{beam}$. The scale is given by the wedge (mJy/beam) at the top. Right: as left but with beam squint corrections. The rms noise is $\sim 105 \mu \mathrm{Jy} /$ beam.

to achieve a more accurate characterization of the squint correction which should lead to improved correction of the effects discussed here as well as of the next order of cross-polarization (leakage) corrections. We hope to report on those results in due course.

Acknowledgements. We thank Peter Napier, Jim Ruff and Ken Sowinski for numerous discussions on the actual characteristics of the (E)VLA antennas, optics and feed systems. We are indebted to Lynn Matthews for her excellent bandpass calibration of the observations of IC 2233 which required the computation and application of low-order polynomial corrections as a function of time. We would like to acknowledge as well useful discussions with Tim Bastian, Sanjay Bhatnagar, Walter Brisken, Jim Condon, Tim Cornwell, Rick Fisher, Frazer Owen and Rick Perley. We are grateful to Bob Sault (the referee) for pointing out an error in our original Eq. (7) and for suggestions that have allowed us to present a shorter, more concise description of our examples and conclusions. 


\section{References}

Abramowitz, M., \& Stegun, I. A. 1964, Handbook of Mathematical Functions, Washington, National Bureau of Standards

Bhatnagar, S., Cornwell, T. J., Golap, K., \& Uson, J. M. 2008, A\&A, submitted Chu, T.-S., \& Turrin, R. H. 1973, IEEE Trans. Antennas Propagat., AP-13, 339 Clark, B. G. 1980, A\&A, 89, 377

Condon, J. J., Cotton, W. D., Greisen, E. W., et al. 1998, AJ, 115, 1693

Cotton, W. D. 1999, in Synthesis Imaging in Radio Astronomy II, ed. R. A. Perley, F. R. Schwab, \& A. H. Bridle, ASP Conf. Ser. , 6, 233

Cotton, W. D. 2008, PASP, 120, 439

Cotton, W. D., \& Uson, J. M. 2007a, EVLA Memo 113,

ftp://ftp.cv.nrao.edu/NRAO-staff/bcotton/Obit/Squint.pdf
Cotton, W. D., \& Uson, J. M. 2007b, A\&A, submitted

Duan, D.-W., \& Rahmat-Samii, Y. 1991, IEEE Trans. Antennas Propagat., 39, 612

Matthews, L. D., \& Uson, J. M. 2008, AJ, 135, 291

Napier, P. J. 1994, MMA Memo, 115, 1

Napier, P. J., \& Gustincic, J. J. 1977, in Digest of the IEEE International Symposium, Stanford: IEEE Antennas and Propagation Society, 452

Rusch, W. V. T., Prata, J. A., Rahmat-Samii, Y., \& Shore, R. A. 1990, IEEE Trans. Antennas Propagat., 38, 1141

Schwab, F. R. 1983, in Indirect Imaging, ed. J. A. Roberts (Cambridge, England: Cambridge University Press), 333

Uson, J. M. 2007, VLA Test Memo 237 